\title{
Accurate Antenna Pattern Modelling for Spaceborne Active Phased Array Antennas
}

\author{
M. Bachmann, M. Schwerdt, B. Döring, C. Schulz \\ Microwave and Radar Institute of the German Aerospace Center (DLR) \\ P.O.-Box 1116, D-82230 Weßling, Germany \\ markus.bachmann@dlr.de
}

\begin{abstract}
In example of measurements performed in space with TerraSAR-X, an approach for an accurate antenna characterization is described. TerraSAR-X was launched in June 2007 and is a highly flexible $\mathrm{X}$-band radar satellite based on an active phased array antenna. Its primary objective is the acquisition of high quality SAR images in a multitude of possible acquisition modes. As this demand is of paramount importance, an accurate characterization of the antenna is a major task, i.e. thousands of different antenna beams being operated have to be determined precisely for compensating the impact of the antenna on SAR data products. Only then, a spaceborne SAR system based on an active phased array antenna can be successfully commissioned. This paper presents the methodology and results derived from different measurements performed for characterizing an active phased array antenna in flight.
\end{abstract}

\section{INTRODUCTION}

Actual radar instruments of a spaceborne SAR systems host an active phased array antenna. It consists of a large number of sub radiation elements each controlled by an individual transmit/receiver module. Thus, adaptive beam forming can be performed allowing a multitude of different operation modes for antenna beam steering and shaping.

The radar instrument of TerraSAR-X with a length of $4.8 \mathrm{~m}$ and a width of $0.7 \mathrm{~m}$ consists of 384 slotted wave-guides arranged in 12 panels in azimuth direction (columns) each with 32 subarrays (rows) [1]. The nominal antenna pointing in elevation is $33.8 \mathrm{deg}$ away from nadir. Right and left looking acquisition is realised by satellite roll manoeuvres. Each individual subarray is driven by transmit-receive modules (TRMs) adjustable in amplitude and phase by applying complex excitation coefficients. This enables beam steering and adaptive beam forming in both azimuth and elevation direction. For the multitude of standard acquisition modes possible on TerraSAR X - like nominal Stripmap, ScanSAR or Spotlight - and the several experimental modes - like quadpol mode, wide band operation or along-track interferometry - more than 10,000 different beams can be commanded.

This great amount of beams and modes is one highlight but also a great challenge of the whole mission. On this, a novel antenna characterization approach has been developed and established based on a precise antenna model and the so called PN gating method [2].

\section{Antenna Model}

The model is primarily used to generate the antenna patterns needed by the processing system to correct for the antenna characteristic of each beam. The antenna characteristic is mapped into the acquired SAR signal as the reflection of the ground is overlaid by the gain pattern of the antenna in range. In azimuth, the pattern is contained in the Doppler spectrum and hence needed for correct Doppler estimation. A second important reason for the antenna model is the optimization of the beam excitation coefficients prior to launch to achieve an optimum performance for the full performance beams in terms of noise equivalent sigma zero and total ambiguity ratio.

The development of the antenna model was driven by three main requirements:

- The great number of more than 10,000 different beams to be calibrated.

- The tight performance requirement of an overall radiometric accuracy goal of better than $1 d B$. From this requirement the accuracy for the antenna model of better than $0.2 d B$ for reproducing the pattern shape and predicting the absolute gain was derived.

- The desired short duration of the commissioning phase.

To ensure these requirements, several steps were realized:

a) As much effort as possible was moved from inorbit tasks to on-ground duties. This includes the accurate measurement of the embedded subarray patterns as well as the validation of the model onground before launch. This was done by comparing modelled patterns with measured patterns. This was successfully performed by Astrium and DLR, showing a first confidence on the excellent performance of the antenna model [3].

b) Different in-orbit calibration techniques for antenna model verification and a sufficiently high amount of measurements, in case of TerraSAR-X over two hundred measurements were executed Therefore, in-orbit verification was performed during the commissioning phase in the first months after launch.

c) To ensure a short commissioning phase, the verified antenna model was used to support the radiometric calibration as well as for pointing calibration [4].

\section{ON-GROUND VALIDATION}

After design and implementation of the antenna model, at first an on-ground validation has been performed. The onground characterisation was realised in two stages. In a first 


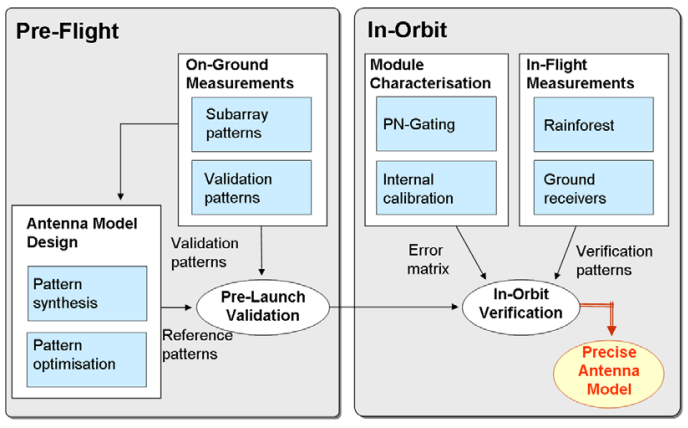

Fig. 1. General antenna model verification approach.

step, the antenna was accurately measured in the Planar Near Field Scanner at and from Astrium GmbH, Germany. In the second step, the correct application of the conventions and input parameters was verified.

For the first step, two kinds of antenna patterns were determined in the Planar Near Field Scanner: The embedded subarray patterns are required as a direct input into the antenna model. For comparison, the patterns of one complete panel or leaf (one third of the whole antenna) was measured.

Due to the dimensions of the whole antenna $(4.8 \mathrm{~m} \times$ $0.7 \mathrm{~m}$ ), the pre-launch validation was performed only up to the stage of one leaf, this is one third of the antenna or four panels in a column. To ensure a valid model even for the complete antenna, the accuracy of the antenna model was tracked through the whole built process of the antenna from individual subarray patterns over the patterns of one panel up to the patterns of the three leaves. Consistent accordance has been found within this development process between the antenna model and the measurements. The measurement in an anechoic chamber is not exactly the same as if the antenna is mounted on the satellite. Although, as there are no parts of the satellite structure rising into the near field of the SAR Antenna, the effects of the satellite and its mounting structure were expected to be small. Additionally, the measurements on satellite level in orbit were expected to be better than the leaflevel measurements as three times more TRMs on the complete antenna provide better statistical distribution for the variation of the TRMs.

For the pre-launch validation, all patterns were measured for both polarisation and at five frequencies. After the measurement, the obtained near field patterns were transformed into the far field using a Fourier transform algorithm. Then, the antenna patterns generated with the antenna model using the embedded patterns were compared to the measured patterns of the complete panel and leaf respectively. The results of this validation on leaf level showed a deviation of less than $\pm 0.2 \mathrm{~dB}$ between the simulated and measured antenna pattern within the $3 \mathrm{~dB}$ main lobe of the beam [5].

After the successful on-ground validation of the antenna model, the correct application of the conventions in the antenna control unit was verified on satellite level. Herefore the complete antenna was already mounted on and connected to the satellite system. Especially the correct handling of the underlying inputs like antenna excitation coefficients, the correct numbering of the antenna elements and the correct steering angle application were successfully tested. Also, the correct update of all changeable antenna parameters like TRM enabling/disabling or excitation coefficients table was verified.

The pre-flight validation proved a very stable and accurate instrument, followed by the successful in-orbit verification as shown in the next chapter.

\section{IN-ORBIT VERIFICATION}

The verification in flight is performed by a comparison between reference pattern derived from the antenna model with those patterns really measured in flight for both the shape within the main-beam and the gain-offset between different beams. This verification of the antenna model is performed with a few selected beams really measured in-flight and can be divided into three main tasks:

- measurements across the rainforest to verify the elevation pattern shape,

- ScanSAR measurements over rainforest to verify the calculated vs. simulated peak-to-peak gain offset between different beams and

- measurements using ground receivers to verify azimuth pattern shape.

\section{A. Antenna Model Verification in Elevation}

In elevation, the simulated relative antenna patterns are compared with estimated patterns. The estimates are extracted from SAR images acquired over rain forest in the Amazon basin, Brazil (see Figure 2a)). Amazon rain forest is a quite homogeneous scatterer and the pattern shape is clearly visible in the SAR raw data. However, as this raw data consist of pulses without any processing, the image processed in the nominal SAR processor is used for pattern estimation. In the processing chain, the accurate position and geometry of the acquisition is determined and annotated after azimuth and range compression. Then the antenna patterns mapped in the image data are corrected with the available modelled reference antenna patterns [5].

For pattern estimation, this pattern correction has to be reversed with the used reference patterns to obtain the original antenna characteristics in the image. Then, the image is freed from disturbances like rivers or de-forested areas using an automatic masking algorithm. The radar image is denoted in beta nought where the backscatter depends on the incidence angle . For the comparison however, the gamma nought has to be derived via the sigma nought using the formula [6]:

$$
\gamma^{0}=\sigma^{0} / \cos (\theta)=\beta^{0} \cdot \tan (\theta)
$$

Finally all azimuth lines are summed up and the pixelelevation angle transformation is applied to obtain the so called Gamma Profile which is a vector of the mean antenna pattern over elevation angle. 


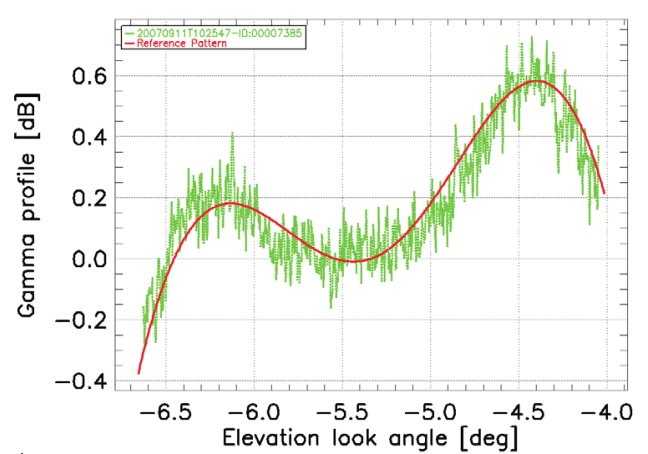

a)

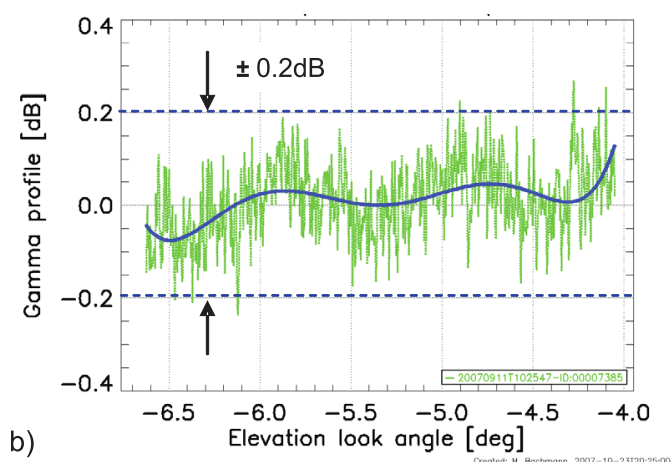

Fig. 2. a) Comparison of the gamma profile (green) derived from a rain forest scene and the reference elevation pattern (red), b) difference between reference pattern and gamma profile, the blue line is a fit of this difference.

An exemplary result is depicted in Figure 2a). The noisy ripple is the gamma profile, which now can be compared to the modelled reference pattern depicted in red.

The described evaluation was performed for a huge number of acquisitions in different beams and polarisations. The results show a quite excellent accordance between the simulated antenna pattern and the measured gamma profiles. The deviation and hence the accuracy of the antenna model is below $\pm 0.2 d B$ peak-peak. This can be seen in Figure 2b) where the deviation between the reference antenna pattern and the estimated pattern is depicted for the exemplary beam. Additionally, by fitting a curve into the profile, a noise-free picture can be obtained.

\section{B. Antenna Model Verification in Azimuth}

Although the algorithm used in the antenna model for calculating the antenna patterns is equal for elevation and azimuth pattern calculation, both directions were verified to ensure the antenna model quality and by this checked for eventual deviations in the underlying components like power dividers or amplifiers.

The verification of the antenna pattern in azimuth direction was performed for transmit pattern only using the DLR ground calibration equipment in form of ground receivers [7] as shown in Figure ??. These receivers record the amplitude of the pulses transmitted by the SAR antenna. Transformed to the antenna elevation angles and corrected by position information, the azimuth antenna pattern is obtained and can

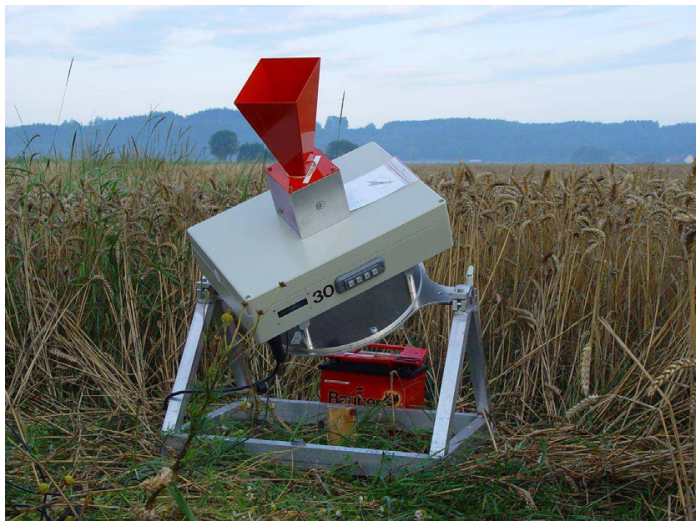

Fig. 3. DLR ground receiver deployed for receiving radar pulses.

be compared to the modelled pattern. The measurements were performed for patterns over the whole specified angular range of the SAR antenna with ground receivers placed at the near, mid and far range within the swaths.
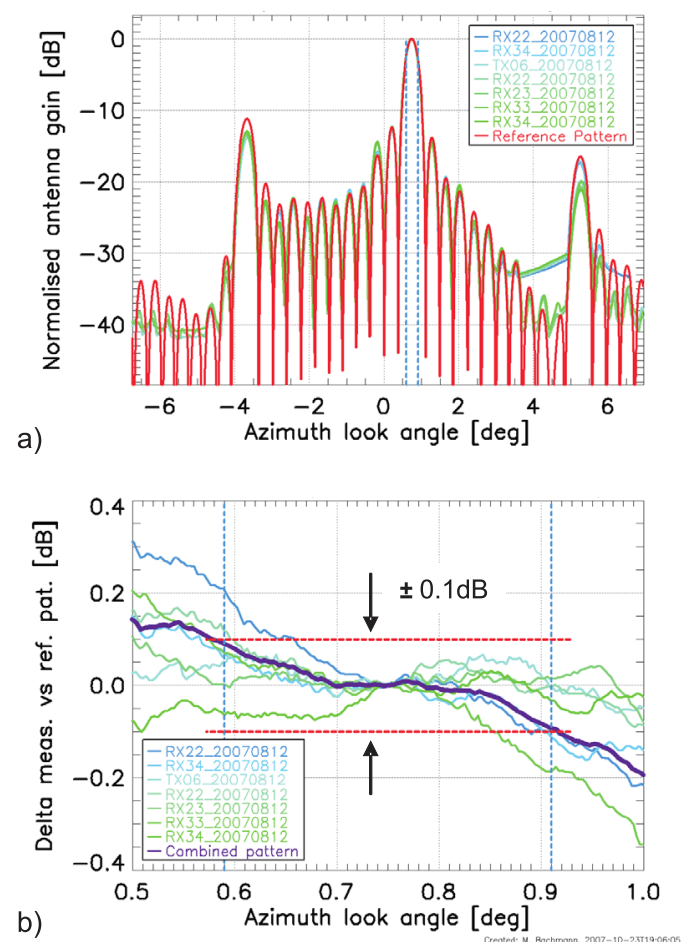

Fig. 4. a) Transmit patterns compared to a reference pattern and b) their deviation.

Figure 4 shows the special case of a double squinted beam. This is a beam steered to angles of $+0.75 \mathrm{deg}$ in azimuth and $16.5 \mathrm{deg}$ in elevation which is at the specified limits for antenna steering. Again, the reference pattern generated by the model is depicted in red, the measured antenna patterns in green to blue. The measured patterns are quite smooth which results from an averaging over several independent pulses. As for elevation, the antenna model verification shows extra-ordinary 
results. The resulting deviation within the $3 d B$ beamwidth for one pass as shown in purple in Figure $4 \mathrm{~b}$ ) is less than $\pm 0.1 \mathrm{~dB}$. This is half of the nominal requirement as only the one-way pattern is measured with ground receivers.

\section{Verification of the Beam-to-Beam Gain Offset}

Besides the verification of the pattern shape, the beamto-beam gain prediction capability of the antenna model is of great importance. First, this is required for the ScanSAR processing, where four different beams with different antenna gains are acquired, corrected with their corresponding pattern and combined into one image. Second, with an appropriate gain prediction, only one Absolute Calibration Factor can be derived for the complete system [8].

The beam-to-beam gain prediction is verified evaluating ScanSAR data. In ScanSAR acquisitions, the beam is switched sequentially from burst to burst between the four neighbouring swaths to get a broader swath width than for normal Stripmap acquisitions. By generating the un-normalised gamma profile for each of the four swaths, the relative gain deviation can be determined. In Figure 5, several of these ScanSAR evaluations for different beams have been combined in one diagram to show the gain prediction over a wide angular region. As the images were acquired over different parts of the rain forest with different vegetation types and hence different backscatter, each complete ScanSAR acquisition was connected to their predecessor within the overlapping regions. The upper part of Figure 5 shows the gamma profiles versus reference pattern, the plot in the middle depicts the deviation between pattern and profile. The lower part shows the deviation between the overlapping regions of two ScanSAR swaths of one acquisition. The results again have an excellent performance of below $\pm 0.2 d B$ peak-peak except one area where a heavy thunderstorm disturbed the acquisition.

By these excellent results verifying a precise antenna model the effort for calibrating and consequently commissioning a complex SAR system can be reduced extremely because only a few selected beams have to be really measured in-flight.

\section{Conclusion}

The results of measurements performed with TerraSAR-X show the suitability of the described approach for characterizing accurately an active phased array antenna in space. This approach is based on an antenna model for generating the reference patterns and providing the gain offset between different patterns. Precise measurements of antenna patterns in orbit better than an accuracy of $\pm 0.2 \mathrm{~dB}$ show the excellent performance of both the antenna model and the TerraSAR$\mathrm{X}$ satellite. Applying furthermore so called notch patterns a knowledge of the antenna pointing down to a few millidegree could be achieved.

This approach contributes not only to the tight performance of spaceborne SAR systems with high accuracy but also to an efficient way of commissioning such complex systems. Hence, this approach is also applicable for the next generation of SAR missions like TanDEM-X or Sentinel-1.
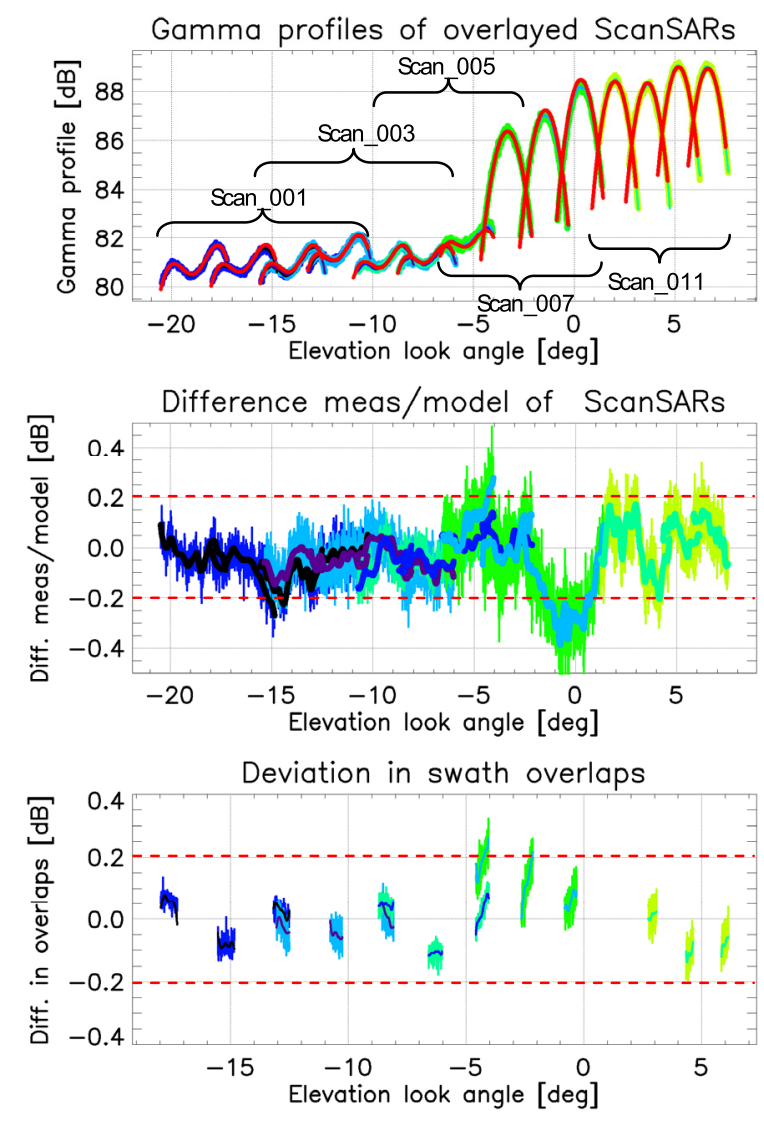

Fig. 5. Beam-to-beam gain verification using ScanSAR images.

\section{REFERENCES}

[1] Stefan Buckreuss, Rolf Werninghaus, and Wolfgang Pitz, "German Satellite Mission TerraSAR-X," in 2008 IEEE Radar Confrerence, Rome, Italy, 2008.

[2] Benjamin Bräutigam, Jaime Hueso Gonzalez, Marco Schwerdt, and Markus Bachmann, "Radar Instrument Calibration of TerraSAR-X," in 7th European Conference on Synthetic Aperture Radar, Friedrichshafen, Germany, 2008.

[3] Markus Bachmann, Marco Schwerdt, Benjamin Bräutigam, Bernhard Grafmüller, Alexander Herschlein, and José Luis Alvarez-Pérez, "The TerraSAR-X Antenna Model Approach," in 2nd International ITG Conference on Antennas, 2007.

[4] Markus Schwerdt, Markus Bachmann, Dirk Schrank, Björn Döring, Benjamin Bräutigam, Jaime Hueso Gonzalez, and Clemens Schulz, "Precise Calibration Techniques for Complex SAR Systems Based on Active Phased Array Antennas," in 2010 Phased Array, Bosten, MA, USA, 2010.

[5] José Luis Alvarez-Pérez, Marco Schwerdt, and Markus Bachmann, "TerraSAR-X Antenna Pattern Estimation by Complex Treatment of Rainforest Measurements," in 26th International Geoscience And Remote Sensing Symposium, Denver, USA, 2006.

[6] H. Laur and J. E. Laycock, "Ers-1 sar antenna pattern estimation," Tech. Rep., ESA-ESTEC, 1994.

[7] Björn Döring, Marco Schwerdt, and Robert Bauer, "TerraSAR-X Calibration Ground Equipment," in WFMN07, Conference of ITG - Commission on Wave Propagation, Dresden, Germany, 2007.

[8] Marco Schwerdt, Benjamin Bräutigam, Markus Bachmann, Björn Döring, Dirk Schrank, and Jaime Hueso Gonzalez, "Final Results of the Efficient TerraSAR-X Calibration Method," in 2008 IEEE Radar Confrerence, Rome, Italy, 2008. 